\title{
Roles of Prefrontal Cortex and Mediodorsal Thalamus in Task Engagement and Behavioral Flexibility
}

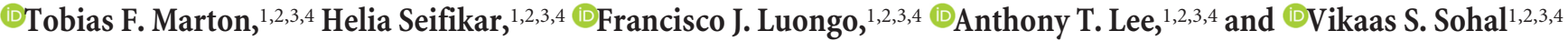 \\ ${ }^{1}$ Department of Psychiatry, ${ }^{2}$ Weill Institute for Neurosciences, ${ }^{3}$ Kavli Institute for Fundamental Neuroscience, and ${ }^{4}$ Sloan-Swartz Center for Theoretical \\ Neurobiology, University of California, San Francisco, San Francisco, California 94143
}

Behavioral tasks involving auditory cues activate inhibitory neurons within auditory cortex, leading to a reduction in the amplitude of auditory evoked response potentials (ERPs). One hypothesis is that this process, termed "task engagement," may enable contextdependent behaviors. Here we set out to determine (1) whether the medial prefrontal cortex ( $\mathrm{mPFC}$ ) plays a role in task engagement and (2) how task engagement relates to the context-dependent processing of auditory cues in male and female mice performing a decisionmaking task that can be guided by either auditory or visual cues. We found that, in addition to auditory ERP suppression, task engagement is associated with increased mPFC activity and an increase in theta band $(4-7 \mathrm{~Hz})$ synchronization between the mPFC and auditory cortex. Optogenetically inhibiting the mPFC eliminates the task engagement-induced auditory ERP suppression, while also preventing mice from switching between auditory and visual cue-based rules. However, mPFC inhibition, which eliminates task engagementinduced auditory ERP suppression, did not prevent mice from making decisions based on auditory cues. Furthermore, a more specific manipulation, selective disruption of mPFC outputs to the mediodorsal (MD) thalamus, is sufficient to prevent switching between auditory and visual rules but does not affect auditory ERPs. Based on these findings, we conclude that (1) the mPFC contributes to both task engagement and behavioral flexibility; (2) mPFC-MD projections are important for behavioral flexibility but not task engagement; and (3) task engagement, evidenced by the suppression of cortical responses to sensory input, is not required for sensory cue-guided decision making.

Key words: cognitive flexibility; decision making; evoked response potential; mediodorsal thalamus; prefrontal cortex

\section{Significance Statement}

When rodents perform choice-selection tasks based on sensory cues, neural responses to these cues are modulated compared with task-free conditions. Here we demonstrate that this phenomenon depends on the prefrontal cortex and thus represents a form of "top-down" regulation. However, we also show that this phenomenon is not critical for task performance, as rodents can make decisions based on specific sensory cues even when the task-dependent modulation of responses to those cues is abolished. Furthermore, disrupting one specific set of prefrontal outputs impairs rule switching but not the task-dependent modulation of sensory responses. These results show that the prefrontal cortex comprises multiple circuits that mediate dissociable functions related to behavioral flexibility and sensory processing.

\section{Introduction}

The rodent medial prefrontal cortex (mPFC) is necessary for tasks that involve switching between different rules to guide be-

Received June 16, 2017; revised Jan. 30, 2018; accepted Feb. 2, 2018.

Author contributions: T.F.M. and V.S.S. designed research; T.M., H.S., and A.T.L. performed research; T.M. and F.J.L. analyzed data; T.F.M. and V.S.S. wrote the paper.

This work was supported by the NIMH (Grants R01 MH100292 and R01 MH106507 to V.S.S. and Grant R25 MH060482), the NIH Office of the Director (Grant DP2 MH100011 to V.S.S.), and an APF-Genentech fellowship to T.M. The authors declare no competing financial interests.

Correspondence should be addressed to Vikaas S. Sohal, University of California, San Francisco, Box 0444, San Francisco, CA 94143. E-mail: vikaas.sohal@ucsf.edu.

T. Marton's present address: San Francisco VA Health Care System, 4150 Clement Street, San Francisco, CA 94121. DOI:10.1523/JNEUROSCI.1728-17.2018

Copyright $\odot 2018$ the authors $\quad 0270-6474 / 18 / 382569-10 \$ 15.00 / 0$ havior (Ragozzino et al., 1999; Birrell and Brown, 2000; Bissonette et al., 2008; Floresco et al., 2008; Durstewitz et al., 2010; Rodgers and DeWeese, 2014; Cho et al., 2015). Indeed, recordings in both primates (Wallis et al., 2001; Johnston et al., 2007; Mante et al., 2013) and rodents (Durstewitz et al., 2010; Rodgers and DeWeese, 2014) have found that prefrontal neurons encode the current rule during task-switching paradigms. At the same time, several studies have described ways in which prefrontal regions modulate sensory responses. In rodents, projections from anterior cingulate cortex, often considered part of the dorsal $\mathrm{mPFC}$, directly modulate visual cortical responses (Zhang et al., 2014). The mPFC also regulates the gain of sensory responses in thalamus (Wimmer et al., 2015). In primates, prefrontal le- 
sions reduce attentional modulation of visual cortical responses (Gregoriou et al., 2014).

Here, we set out to identify ways in which the $\mathrm{MPFC}$ regulates sensory responses and rule switching during a task in which mice make decisions using either auditory or visual cue-based rules. In particular, responses within the auditory cortex $(\mathrm{AC})$ are smaller when auditory stimuli are presented in the context of a behavioral task compared with a passive, task-free condition (Otazu et al., 2009; Fritz et al., 2010). This process, sometimes called "task engagement," may reflect an increase in the responses of inhibitory, but not excitatory, neurons in auditory cortex (Kuchibhotla et al., 2017). Cholinergic input is necessary for this phenomenon, but it remains unclear whether other brain regions such as the mPFC might be involved.

We explored whether the mPFC is involved in the task-related suppression of cortical sensory responses and how this phenomenon relates to other functions of the MPFC, specifically switching between auditory and visual cue-based rules for making decisions. We studied these questions using a combination of optogenetics and electrophysiology in freely behaving mice. We find that the task-related suppression of auditory cortical responses depends on top-down regulation by the mPFC. At the same time, we show that this task-related response suppression does not appear to be necessary for mice to make decisions based on auditory cues. Furthermore, output from the mPFC to mediodorsal (MD) thalamus is necessary for switching between auditory and visual rules, but not for the task-related suppression of auditory responses. These results show that the MPFC contributes to both behavioral flexibility and task engagement but does so through distinct pathways.

\section{Materials and Methods}

Subjects. All experiments were conducted in accordance with procedures established by the Administrative Panels on Laboratory Animal Care at the University of California, San Francisco. Ten- to 16-week-old C57BL/6 mice of either sex (51 mice total; 41 males, 10 females) were purchased from Simonsen Laboratories and group housed under a $12 \mathrm{~h}$ light/dark cycle with freely available food and water. For experiments, mice were given injections of virus and implanted with EEG electrodes as described below and allowed to recover for 5 weeks under standard group-housed conditions. Mice were then singly housed, food deprived, and maintained at $85 \%$ of original body weight before engaging in the behavioral task.

Viral injections. Mice received stereotactic injections of $750 \mathrm{nl}$ of adeno-associated virus type 5 (AAV5) expressing the inhibitory opsin Arch3.0 and eYFP (or eYFP alone) under control of the human synapsin promoter (AAV5-synapsin-Arch3.0-eYFP or AAV5-synapsin-eYFP, UNC Viral Core) bilaterally into $\mathrm{mPFC}$ ( $1.7 \mathrm{~mm}$ AP, $0.3 \mathrm{~mm} \mathrm{ML},-2.75$ $\mathrm{mm}$ DV relative to bregma). Mice recovered for 5 weeks after injection to allow time for viral expression and axonal transport of Arch3.0 and eYFP. Appropriate anatomical expression was confirmed after the experiment through histological analysis and visualization of eYFP expression.

EEG implantations. Conductive stainless steel screws that served as recording electrodes (Pinnacle Technology) were implanted intracranially over bilateral $\mathrm{mPFC}(1.7 \mathrm{~mm} \mathrm{AP}, 0.3 \mathrm{~mm} \mathrm{ML},-1.75 \mathrm{~mm}$ DV relative to bregma), bilateral motor cortex ( $0 \mathrm{~mm} \mathrm{AP}, 1 \mathrm{~mm} \mathrm{ML},-0.5 \mathrm{~mm} \mathrm{DV})$, and bilateral auditory cortex ( $-2.0 \mathrm{~mm} \mathrm{AP}, 2.25 \mathrm{~mm} \mathrm{ML},-1.75 \mathrm{~mm} \mathrm{DV}$ relative to bregma). A common electrode wired to each auditory cortical electrode was implanted in the bony calvarium overlying the cerebellum ( $-5.0 \mathrm{~mm} \mathrm{AP,} 0 \mathrm{~mm} \mathrm{ML},-1.75 \mathrm{~mm}$ DV relative to bregma). These screws were attached to a head mount using conductive wire, dental cement was used to secure the head mount, and animals were allowed to recover for 5 weeks before recording sessions were initiated.

Dual implant surgeries. To generate mice for optogenetic manipulation of mPFC while recording from AC, viral construct injection (AAV5synapsin-Arch3.0-eYFP or AAV5-synapsin-eYFP, UNC Viral Core) was performed as described above. After bilateral viral injection into mPFC, dual fiber-optic cannulae (Doric Lenses) were implanted intracranially over the viral injection sites (1.7 $\mathrm{mm} \mathrm{AP,} 0.3 \mathrm{~mm} \mathrm{ML},-2.25 \mathrm{~mm} \mathrm{DV}$ relative to bregma). EEG electrode implantation over AC was then performed as described above. For callosal terminal inhibition experiments, mice were given injections of viral constructs unilaterally in mPFC, and a single fiber-optic cannula was placed in the $\mathrm{MPFC}$ in the opposite hemisphere as described above. For mPFC-MD terminal inhibition experiments, viral constructs were injected bilaterally into mPFC and dual fiber-optic cannulae (Doric Lenses) were implanted intracranially over MD bilaterally $(-1.3 \mathrm{~mm} \mathrm{AP,} 0.5 \mathrm{~mm} \mathrm{ML},-3.0 \mathrm{~mm} \mathrm{DV})$. In all cases, mice were allowed 5 weeks of recovery time to allow for viral expression.

EEG recording and analysis. Differential EEG was recorded at $2 \mathrm{kHz}$ from mice implanted with EEG electrodes (Pinnacle Technology) continuously during behavior. The mPFC signal was the difference between left and right prefrontal electrodes. The AC signal was generated for each hemisphere as the difference between the AC electrode and the common electrode placed over cerebellum. To compute power during the attention-shifting task, we computed the average power across various frequency bands during the $8 \mathrm{~s}$ window from when the animal was placed in the cage until bowl selection, and we compared within animals the average power during a $30 \mathrm{~s}$ baseline period before the experiment in which the animal was at rest (measured in log units), using the spectrogram function in Matlab (Mathworks).

Magnitude squared coherence measurements were computed in Matlab using the function mscohere, with an $\mathrm{fft}$ window size of 512 samples and $50 \%$ overlap between windows. Coherence was normalized by subtracting a noise floor determined as the coherence between two surrogate signals that had randomized phases (Prichard and Theiler, 1994). Coherences reported represent the average coherence across electrodes in $10 \mathrm{~s}$ segments in 10 trials either before or after learning.

Auditory evoked response potentials. Mice were presented with a continuous stimulus train consisting of $6 \mathrm{kHz}$ tones $(0.5 \mathrm{~s}$ duration, $1 \mathrm{~s}$ interstimulus interval) at $80 \mathrm{~dB}$ during either passive listening conditions (200 tone reps total) or in the context of the attention-shifting task (180220 tone reps per 10-trial block) while continuous EEG was recorded. Tones were driven by a laptop and synchronized with a TTL pulse that was sent to the EEG apparatus through a Cedrus Stimtracker (https:// www.cedrus.com/stimtracker/) to timestamp the EEG recording with the times of tone presentations. Specifically, the computer-generated audio control signal is sent directly to the StimTracker, which simultaneously transmits the control signal to speakers and generates a TTL pulse at tone onset. We are confident in the temporal precision of this approach based on two observations. First, we were able to reliably generate evoked response potentials (ERPs) using a similar number of tone presentations compared with previously published studies examining the ERP N1 component in mice (Featherstone et al., 2012). Specifically, each trial contained $\sim 8-12$ tone repetitions, and auditory ERPs were based on 10 trials, i.e., $\sim 80-120$ tone repetitions. Second, the N1 component reproducibly occurred between 40 and $50 \mathrm{~ms}$ across trials and animals, consistent with the temporal evolution of the N1 in mice described by Amann et al. (2010). Any significant “jitter" in relative timing between the tone and TTL would have resulted in (1) less reliable ERP waveforms and (2) far less fidelity in the temporal onset and evolution of ERP components such as the N1.

To generate the ERP, raw EEG signals from each AC electrode were first high-pass filtered at $8 \mathrm{~Hz}$ with a first-degree Butterworth filter to eliminate continuous low-frequency oscillations that perturbed ERP waveform and then notch filtered at $60 \mathrm{~Hz}$. ERPs were then generated by averaging the EEG signal from $50 \mathrm{~ms}$ before $150 \mathrm{~ms}$ after each tone onset across all tone repetitions and trials of interest. ERP waveforms were assessed for consistency and appropriate temporal evolution compared with previous literature (Amann et al., 2010). The ERP N1 was identified as the negative-deflecting waveform evolving after the initial poststimulus positivity between 30 and $80 \mathrm{~ms}$. ERP N1 amplitude was calculated as the minimum of the N1 waveform (i.e., the most negative point) and averaged across both electrodes. For animals in which one of the two AC electrodes did not generate a satisfactory ERP waveform (electrical short, excessive noise, etc.), the ERP N1 amplitude was generated from the 
satisfactory electrode. Comparison of ERP amplitudes between conditions was evaluated using parametric statistics (ANOVA, two-tailed $t$ test) as the data were normally distributed.

In vivo optogenetic stimulation. Stimulation of Arch during awake behavior was performed using a $532 \mathrm{~nm}$ green laser (OEM Laser Systems) that was coupled to the dual fiber-optic cannulae (Doric Lenses) through a 200- $\mu \mathrm{m}$-diameter dual fiber-optic patchcord with a guiding socket (Doric Lenses) and a $1 \times 2$ intensity division fiber-optic rotary joint (Doric Lenses), adjusted such that the light power was $\sim 5.0 \mathrm{~mW}$ total power for both fibers (i.e., $\sim 2.5 \mathrm{~mW}$ per side) when performing optical inhibition of neuronal cell bodies. Total power of $2.5 \mathrm{~mW}$ was used for unilateral cell-body inhibition, and $5.0 \mathrm{~mW}$ per fiber was used to silence terminals. Light stimulation was constant throughout the period of illumination.

Auditory-visual rule-switching task. Mice were singly housed before starting the experiment and food deprived to $85 \%$ of initial body weight over several days. For all experiment days, mice were first connected to the EEG apparatus and fiber-optic cord (if applicable); then, a $30 \mathrm{~s}$ baseline recording in the holding cage was performed. On the passive listening day, mice were then manually shifted between the adjacent holding cage and the home cage where the speakers, lights, and food reward bowls were set up as shown in Figure $1 A$. On the "passive listening" day, mice were exposed to 10 tone repetitions in the home cage (with both light cues on), then shifted back to the holding cage for 30 s so as to roughly simulate the experimental conditions of the task day. This was performed 20 times to achieve 200 tone repetitions total. The next day, mice were habituated to the bowl digging aspect of the task: a small peanut butter chip was buried in the sand of one of the two bowls as shown in Figure $1 \mathrm{~A}$. Mice were trained to dig in one of the two bowls to get a food reward. Mice learned to quickly dig in each bowl to find the food reward within 10 training trials. No auditory or light stimuli were presented during the bowl habituation day. On the task day, mice were shifted from the holding cage to the home cage and directed to the rewarded bowl by either the auditory or light cue. Incorrect trials in which the mouse picked the unrewarded bowl resulted in a $2 \mathrm{~min}$ "time out" punishment in the holding cage. Mice learned to associate the auditory cue with the rewarded tray, and a total of 20 "auditory cue" trials were performed; then, optogenetic manipulations were performed for the next 10 auditory trials, followed by 20 "light cue" trials. If mice were unable to reach $80 \%$ performance criteria ( 8 of 10 last trials correct) during the initial 20 auditory cue trials, the experiment was stopped and the trials were discarded from any experimental analysis. We found $\sim 40 \%$ of mice were able to learn the initial auditory association under these conditions. No other animals were excluded, i.e., all mice that learned the auditory rule were able to switch to a visual rule. The numbers for every plot and statistical test are presented in the figure legends. During the rule switch to the light cue, error types were defined as either perseverative (continues to follow the old auditory rule) or random (follows neither rule). Nonparametric statistics (Wilcoxon $U$ test) were used to compare task conditions with respect to error type as the data were not normally distributed.

Experimental design and statistical analysis. The numbers for each experiment are included in the figure legends. Specific statistical tests used and descriptive statistics are explained in Results. For each experiment, there was only one endpoint (either ERP amplitudes or percentage of trials correct), and we simply performed pairwise comparisons between samples from each group. Nonparametric statistics were used as noted when data were not distributed normally. ANOVAs and nonparametric statistics were run using statistical software (SPSS); $t$ tests and $t$ statistics were generated in Excel. In Figure 4, $C$ and D, we observed clear separation in the data with $n=4$, following the same pattern as in the analogous experiment described in Figure 3, $C$ and $D$, and therefore reported the results using paired $t$ tests in this case.

\section{Results}

Using auditory cues to guide behavior leads to suppression of the auditory ERP

In this study, we used an audiovisual switching task in which mice use auditory or light cues to guide them to a rewarded food bowl
(Fig. 1A). On an initial passive listening day, mice were exposed to tone repetitions and light stimuli outside the context of any task, and auditory ERPs were recorded using intracranial EEG electrodes implanted over each AC (Fig. 1A). The auditory ERP (Fig. 1C, black trace), generated by averaging over 200 tone repetitions, conforms to a well established waveform, comprising initial upward and downward deflections at 20 and 40 ms consistent with the early P1 and N1 components commonly described in the human EEG literature (Amann et al., 2010).

After a subsequent day of habituation to the mechanics of the task, mice performed the audiovisual switching task as follows. On each trial, the mouse is presented with two bowls on either side (left or right) of its home cage. Only one bowl contains a hidden food reward. Behind each bowl is a speaker and light source. On each trial, exactly one speaker produces a train of tones, and one light is illuminated. The relationship between the auditory (speaker $\mathrm{ON}$ ) and visual (light $\mathrm{ON}$ ) cues is random, i.e., on $50 \%$ of the trials the activated speaker and light are on the same side; on the remaining trials they are on opposite sides (see Fig. $1 \mathrm{~A}$ for all possible trial types). During the initial portion of the task ("auditory rule"), the activated speaker is always located behind the rewarded bowl. Thus, during the auditory rule portion of the task, the activated light will be behind the rewarded bowl $50 \%$ of the time, i.e., the light cue was irrelevant to task outcome and could be ignored. After mice reached a performance criterion for the auditory rule ( $80 \%$ correct during a 10 trial block), the rule was switched such that the light cue now indicated the location of reward ("visual rule") and the auditory cue could be ignored. Mice learned both auditory and visual rules quickly and could readily switch between them (Fig. 1B). Because the auditory and visual cues were on the same side for $50 \%$ of trials, and on opposite sides for the other $50 \%$, the fact that mice are correct on $>80 \%$ of trials after the switch from an auditory to a visual rule indicates that they are, in fact, using the visual cue, not just going to the side opposite the auditory cue.

During the auditory rule portion of the task, the N1 component of the auditory ERP was suppressed relative to the passive listening phase (Figs. $1 C, D ; t_{(11)}=3.149, p=0.00925$, paired $t$ test). In a separate cohort of mice, we confirmed that changes in ERP amplitudes across days were not simply attributable to repeated exposure to the tones, as there was no significant change in $\mathrm{N} 1$ amplitude across 2 consecutive days of passive listening (Fig. $1 E$; n.s., paired $t$ test). Previous studies have shown that when only a single auditory cue is relevant to task performance, then AC responses are suppressed during task engagement compared with passive listening (Otazu et al., 2009; Fritz et al., 2010), possibly reflecting changes in the cortical signal-to-noise ratio optimizing detection of the task-relevant auditory cue. Indeed, in our audiovisual switching task (which uses a single auditory cue), we find suppression of the AC response to task-relevant versus passively presented auditory cues. Thus, this suppression represents a physiologic biomarker of task engagement that may reflect the top-down modulation of sensory processing.

\section{Performance of the rule-switching task increases mPFC gamma power and engages an MPFC-AC neural network}

Next, we sought to further characterize the neural networks recruited by this task. First, we assayed changes in $\mathrm{MPFC}$ power during the auditory rule portion of the task (Figs. $1 A, 2 A$ ). Learning of the auditory rule elicited changes in MPFC activity, specifically an increase in power within the upper portion of the gamma-frequency band (62-120 Hz; Fig. $2 A ; Z=-2.197, p=$ 0.028 , paired-sample Wilcoxon signed rank test). Similar to pre- 
A

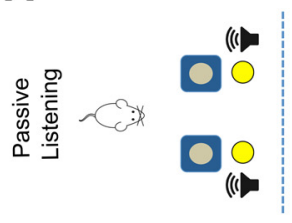

证离<smiles>[BH3-][Hg]</smiles>

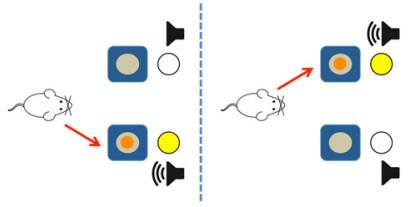

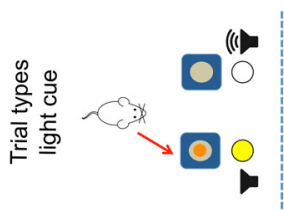
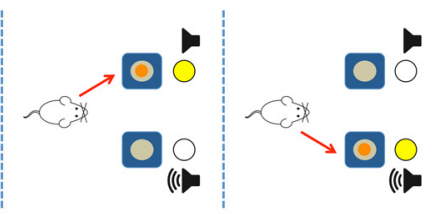

C

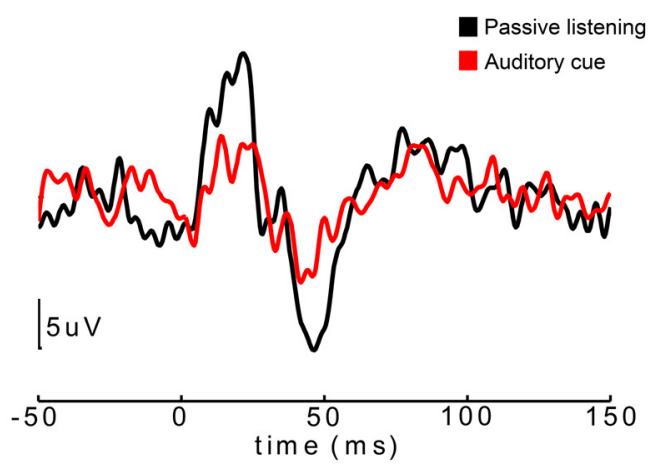

E

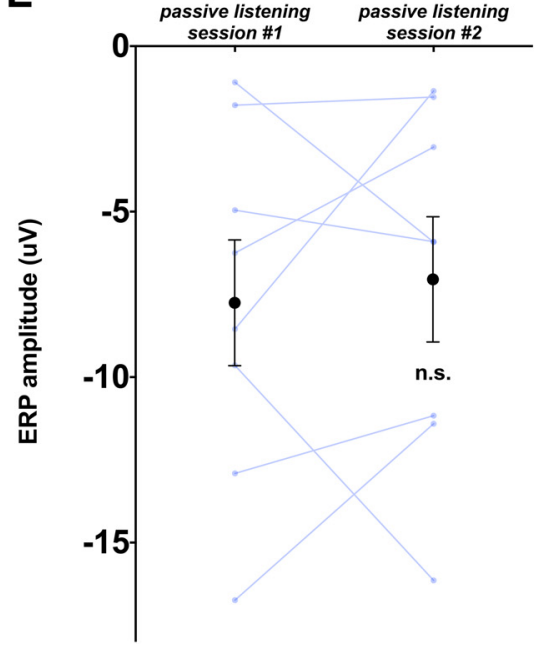

B

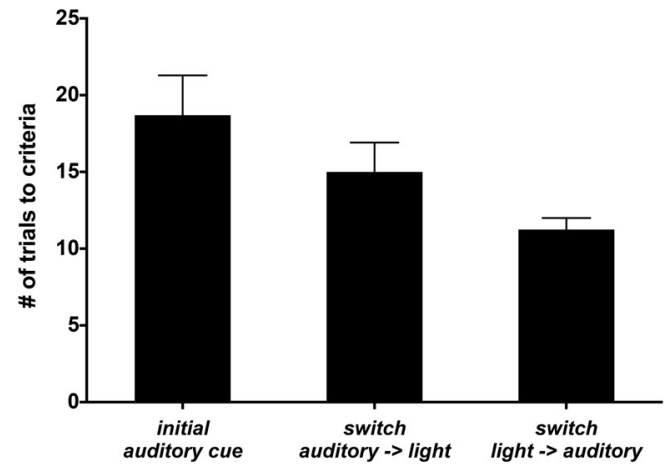

D

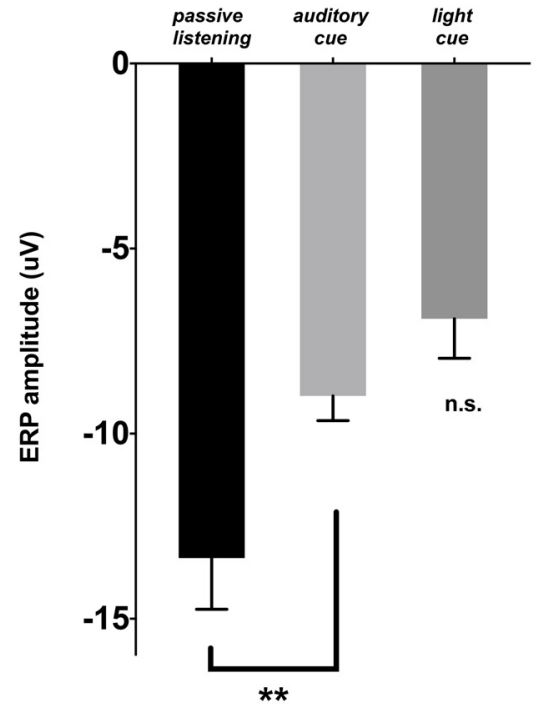

Figure 1. Selective attention to auditory cues elicits suppression of the auditory ERP N1. $\boldsymbol{A}$, Auditory-visual rule-switching task conditions. In passive listening, tone and light stimuli are presented, but neither tray contains a food reward. In the auditory rule condition, tones are played from the speaker on the same side as the rewarded food tray. The position of the light stimulus varies randomly between the rewarded (congruent with auditory stimulus) and unrewarded (incongruent with auditory stimulus) food trays, as shown in the four possible trial types. In the visual rule condition, once mice reach a criterion of 8 of 10 trials correct during the auditory rule portion of the task, the rewarded tray becomes paired with the light stimulus, and the location of the tones varies randomly between the rewarded (congruent) and unrewarded (incongruent) tray, as shown in the four possible trial types. B, Mice rapidly reached (Figure legend continues.) 
A

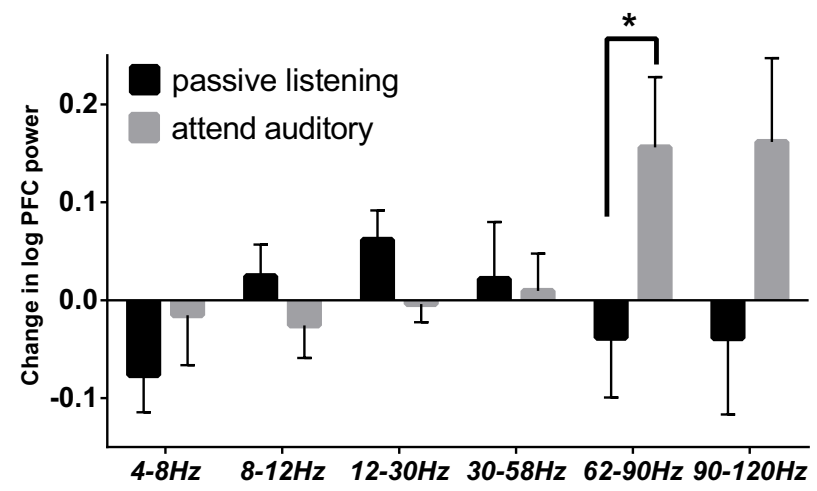

C

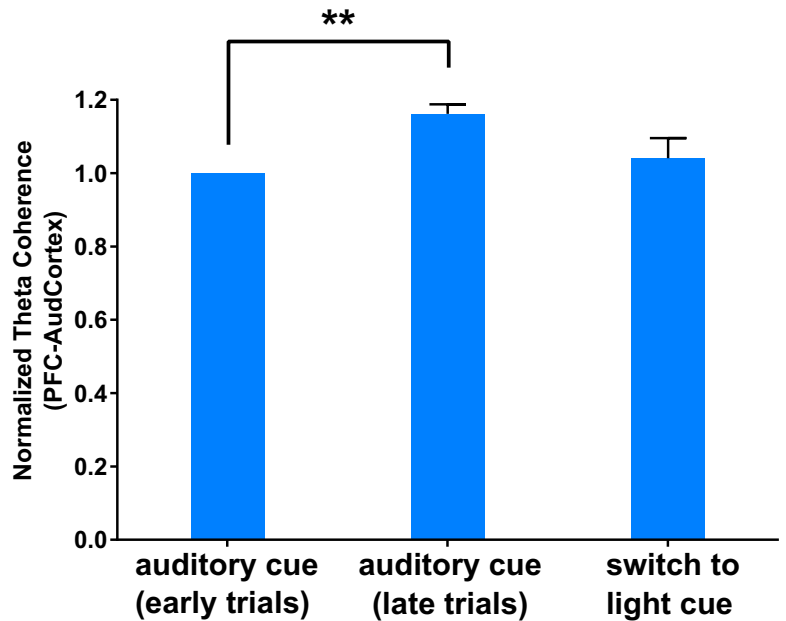

B

\section{Inter-electrode coherence \\ real data shuffled null}
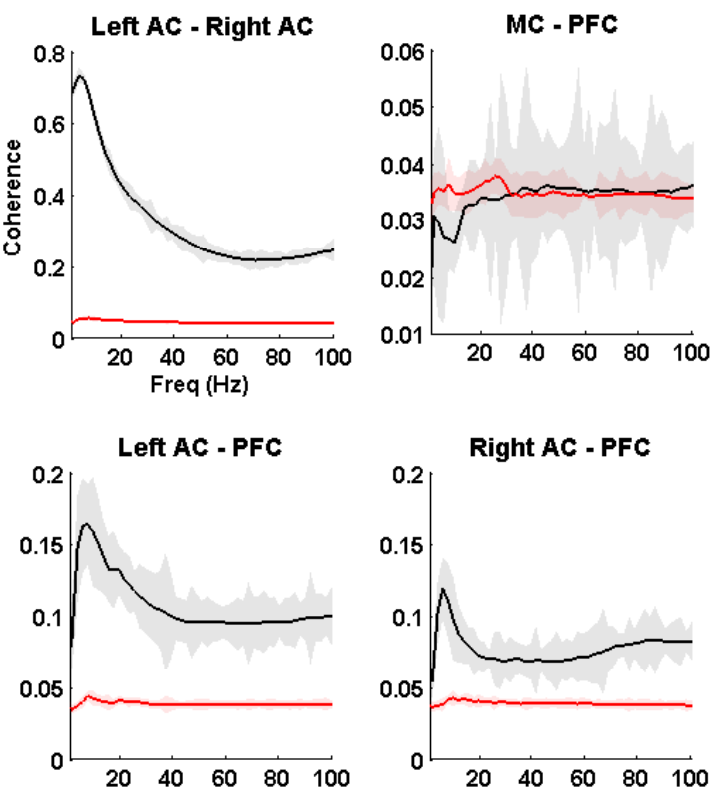

Left AC - MC
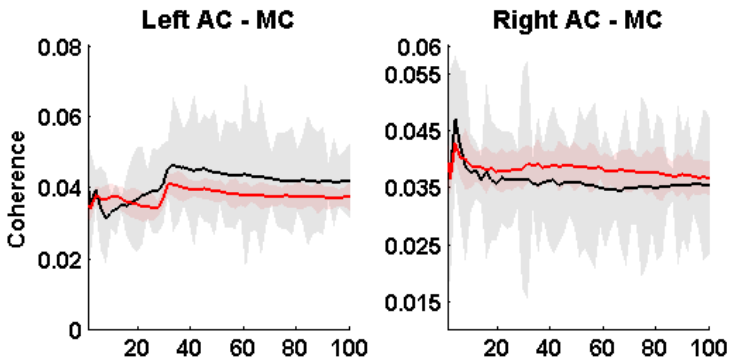

Figure 2. $\mathrm{mPFC}$ gamma power and $\mathrm{mPFC}-\mathrm{AC}$ theta coherence increase during auditory cue-guided behavior. $\boldsymbol{A}$, mPFC power in the gamma band ( $60-120 \mathrm{~Hz}$ ) increases during the auditory rule portion of the task compared with passive listening $(n=7) .{ }^{*} p<0.05 . \boldsymbol{B}$, Coherence versus frequency for all electrode pairs (black) versus boot-strapped data sets (red). Gray shading indicates $+/-1$ SEM. Electrode pairs between PFC and AC show peak coherence in the theta (4-7 Hz) band. $C$, Theta coherence during early auditory rule trials (performance below $80 \%$ correct), late auditory rule trials (at least 8 of 10 trials correct), and visual rule trials (normalized to the coherence during the early attend auditory trails). There is a significant increase in $\mathrm{mPFC}-\mathrm{AC}$ theta coherence as mice learn the auditory rule $(n=6) .{ }^{* *} p<0.01$.

vious studies of other rule-switching tasks in our laboratory (Cho et al., 2015), this simply provides evidence that the mPFC is engaged by our task (additional evidence for a role of the mPFC in this task comes from optogenetic inactivation experiments described below). Having found mPFC recruitment as well as

(Figure legend continued.) the performance criterion (8 of 10 trials correct) while shifting between auditory cue and light cue-based rules $(n=10)$. C, Auditory ERPs in one mouse generated from tones presented during passive listening (black) or the auditory rule condition (red) demonstrate suppression of the ERP N1 (negativity at $40-50 \mathrm{~ms}$ ) during auditory cueguided behavior. D, Quantification of the ERP $N 1$ amplitude during passive listening versus the auditory rule versus visual rule task conditions $(n=12)$. ${ }^{* *} p<0.01$. E, ERP N1 amplitudes do not change during 2 consecutive days of passive listening to the auditory cue (no task engagement; $n=8$ ). changes in auditory ERPs during the task, we next assayed the extent to which the MPFC and AC form a functional network during the auditory rule portion of the task. Coherence between $\mathrm{mPFC}$ and $\mathrm{AC}$ electrodes during the auditory portion of the task was significantly elevated, compared with randomized bootstrapped data, over a broad frequency range, and showed a consistent peak in the theta-frequency range (4-12 Hz; Fig. 2B). Notably, neither the mPFC nor the AC showed significant coherence (relative to randomized data) with electrodes placed in the motor cortex (Fig. $2 B$ ). To determine whether mPFC-AC theta coherence changes as a function of the task, we calculated the maximal theta coherence value during "early" attend auditory trials (the first 10 trials, i.e., before auditory rule acquisition) versus "late" ones (the last 10 trials, i.e., after auditory rule acquisition). We found that peak theta coherence increased from early to late trials of the auditory rule portion of the task, suggesting 
that interactions between the $\mathrm{mPFC}$ and $\mathrm{AC}$ correlate with task acquisition (Fig. $2 C ; t_{(5)}=13.786, p=0.00003$, paired $t$ test).

\section{Optogenetic inhibition of mPFC disrupts auditory ERP modulation and prevents audiovisual rule switching}

Based on our observations that task engagement recruits increases in $\mathrm{mPFC}$ gamma power and that the $\mathrm{MPFC}$ and $\mathrm{AC}$ form a task-dependent functional network, we hypothesized that optogenetic inhibition of mPFC might disrupt task performance and/or the task-dependent modulation of the auditory ERP. To test this hypothesis, we injected AAV5-synapsin-Arch3.0-YFP into bilateral $\mathrm{mPFC}$ and placed electrodes over $\mathrm{AC}$ as described previously (Fig. 3A). After the mice performed 20 attend auditory trials under native conditions, we delivered continuous $530 \mathrm{~nm}$ light to inhibit the mPFC while the mouse performed an additional 10 attend auditory trials, followed by a switch to the visual cue-based rule (Fig. 3B).

We found that optogenetic inhibition of mPFC did not affect behavioral performance based on the recently acquired auditory rule, but it did completely prevent the animal from switching to the visual rule (Fig. $3 C ; Z=-3.18, p=0.0014$, Mann-Whitney $U$ test). In particular, mPFC inhibition elicited predominately perseverative errors in which the animal continued to make decisions based on the auditory cue despite poor task performance (Fig. 3D; $Z=-3.18, p=0.0014$, Mann-Whitney $U$ test). Importantly, the effects of Arch-mediated mPFC inhibition were fully penetrant in every animal, i.e., there was no overlap in performance during the switch (measured either by the percentage of trials correct or the number of perseverative errors) between the Arch-expressing animals and the animals without stimulation. All the animals without stimulation were able to switch successfully between the auditory and visual cue-based rules, whereas none of the Arch-expressing animals that received light stimulation were able to do so.

In this context, we performed a small number of additional control experiments using animals that received light stimulation but expressed eYFP only (no Arch). The numbers for these experiments were relatively small because they were done simply to confirm that the Arch stimulation-induced phenotypes (which were completely penetrant in Arch-expressing animals) were not present in animals that received light but expressed YFP instead of Arch. Indeed, control mice that received light, but which expressed eYFP only (no Arch), performed identically to control animals that did not receive stimulation and did not exhibit the profound phenotypes that light elicited in Arch-expressing mice (Fig. 3C,D).

Furthermore, mPFC inhibition was associated with an increase of the N1 component of the auditory ERP (Fig. $3 E, F$ ). Importantly, this increase was task dependent, as mPFC inhibition had no effect on the auditory ERP during passive listening to tones (Fig. 3F; $F_{(2,44)}=9.1, p=0.002$, ANOVA; laser ON vs OFF for Syn-Arch group: $t_{(5)}=5.197, p=0.0034$, paired $t$ test; WT vs Syn-Arch: $t_{(5)}=3.652 p=0.0147$, paired $t$ test). Again, light delivery had no effect in eYFP-expressing control mice (Fig. $3 F$ ). Thus, mPFC inhibition disrupts modulation of sensory processing, specifically by attenuating the suppression of the N1 component of the auditory ERP. There was a notable temporal dissociation between the behavioral and electrophysiological consequence of mPFC inhibition. Intriguingly, changes in the auditory ERP occurred during trials 21-30 of the attend auditory portion of the task, which the animal continued to perform with high accuracy, whereas behavioral deficits only emerged after the subsequent switch to a visual rule. Notably, the effect of mPFC inhibition to disrupt ERP suppression (i.e., to increase the ERP) was still present after the switch to a visual rule (laser ON SynArch group vs no stimulation group: $F_{(1,10)}=6.18, p=0.032$, ANOVA; laser ON Syn-Arch vs Syn-YFP group: $t_{(8)}=2.84, p=$ 0.02 , unpaired $t$ test).

\section{Inhibition of mPFC projections to MD thalamus disrupts audiovisual rule switching but not ERP modulation}

Prior studies have identified an important role for MD thalamus in flexible goal-directed behavior in other paradigms (Parnaudeau et al., 2013), and PFC-MD interactions are impaired in both schizophrenia and high-risk populations (Woodward et al., 2012; Anticevic et al., 2015). We therefore hypothesized that projections from $\mathrm{mPFC}$ to MD thalamus may mediate some functions of the MPFC in rule switching and/or ERP suppression.

To test this, we injected AAV5-synapsin-Arch3.0-YFP into mPFC and implanted optical fibers over MD thalamus bilaterally. After waiting 5 weeks for expression of Arch3.0 in terminals in the MD thalamus, we once again tested mice on the audiovisual rule-switching task (Fig. $4 A, B$ ). Using Arch to disrupt the transmission of signals from mPFC to MD thalamus resulted in the same inability to switch from auditory to visual rules observed after bilateral mPFC inhibition (Fig. $4 C, D$; performance: $t_{(3)}=$ 60.4, $p=0.00001$, paired $t$ test; errors: $t_{(3)}=47.92, p=0.00002$, paired $t$ test). Also, as in experiments with bilateral mPFC inhibition, performance during the auditory rule portion of the task remained intact (Fig. 4C). However, unlike bilateral mPFC inhibition, bilateral inhibition of mPFC-MD projections did not affect the auditory ERP (Fig. $4 E$; n.s., paired $t$ test). Finally, we again confirmed that the fully penetrant inability to switch to a visual cue-based rule in animals that received light and expressed Arch was not present in mice that received light but expressed eYFP only (no Arch; Fig. 4C,D).

\section{Discussion}

We have shown that the $\mathrm{mPFC}$ is necessary for switching between rules based on different sensory cues (Cho et al., 2015). Other studies have shown that task engagement modulates responses to sensory cues (Otazu et al., 2009; Fritz et al., 2010; Kuchibhotla et al., 2017). Here we show that the mPFC is also necessary for the task engagement-induced modulation of sensory responses but that this function and rule switching depend on distinct sets of prefrontal outputs. Furthermore, when task-induced ERP changes were eliminated by inhibiting $\mathrm{mPFC}$, mice continued making decisions based on auditory cues, with no decrement in their performance, demonstrating a dissociation between ERP suppression related to task engagement and the ability to use a specific sensory cue for decision making.

\section{Prefrontal-thalamic pathways for behavioral flexibility}

Some studies suggest that increases in ERP amplitude contribute to behavioral flexibility. Specifically, Wimmer et al. (2015) studied a task in which mice perform cued switching between auditory and visual cue-based rules on a trial-by-trial basis. In that study, attention to a visual rule was accompanied by increased visual ERPs in the lateral geniculate nucleus. Furthermore, in the study by Wimmer et al. (2015), disrupting this ERP increase (by exciting or inhibiting thalamic reticular neurons) also interfered with task performance. Three key differences between our task and that of Wimmer et al. (2015) may explain why we observed task-dependent ERP suppression and a dissociation between the modulation of sensory responses and behavioral flexibility. 
A

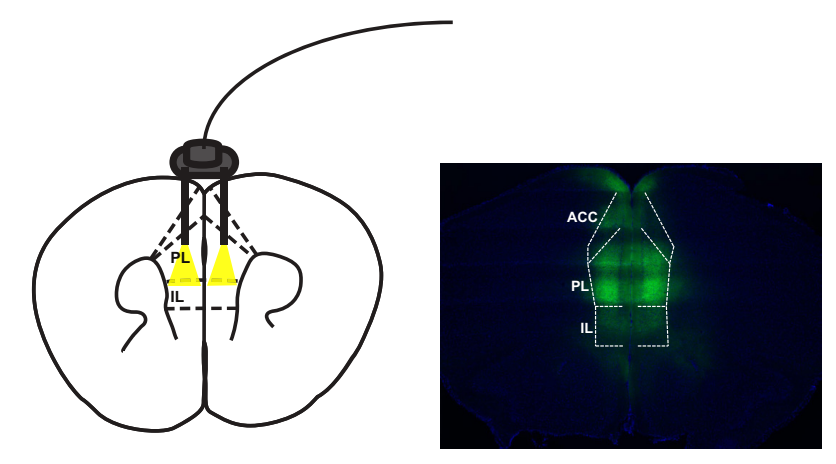

C

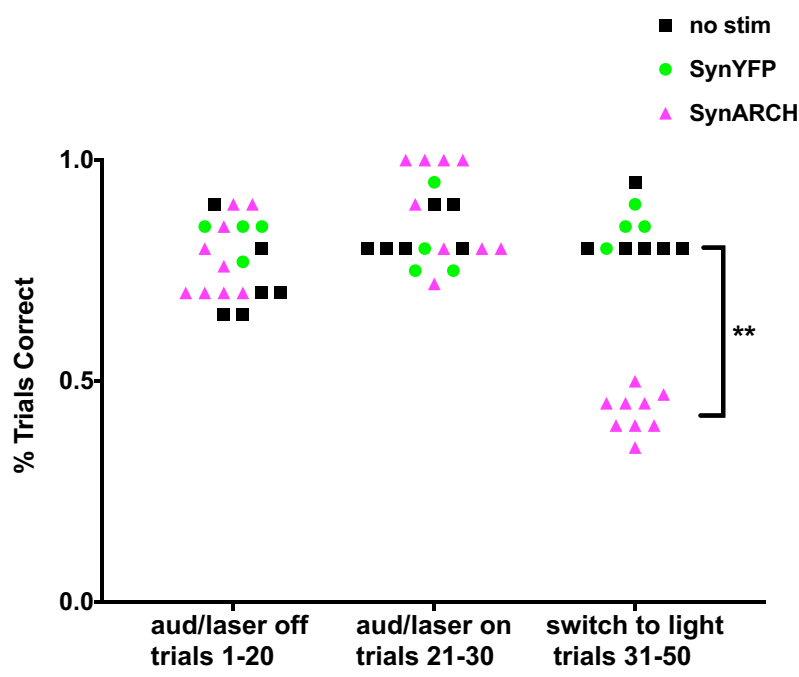

E

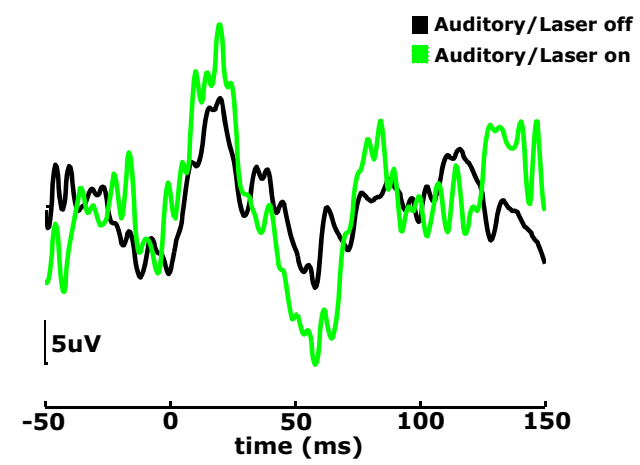

B

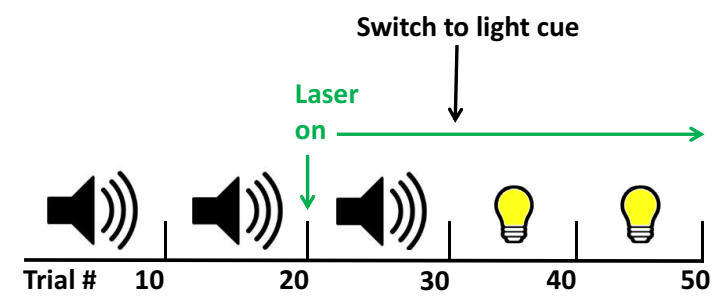

D

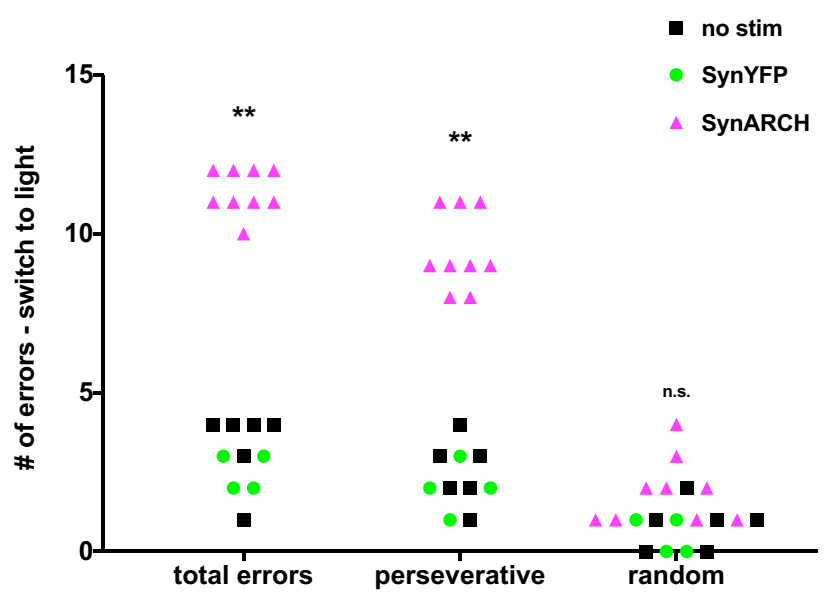

$\mathbf{F}$

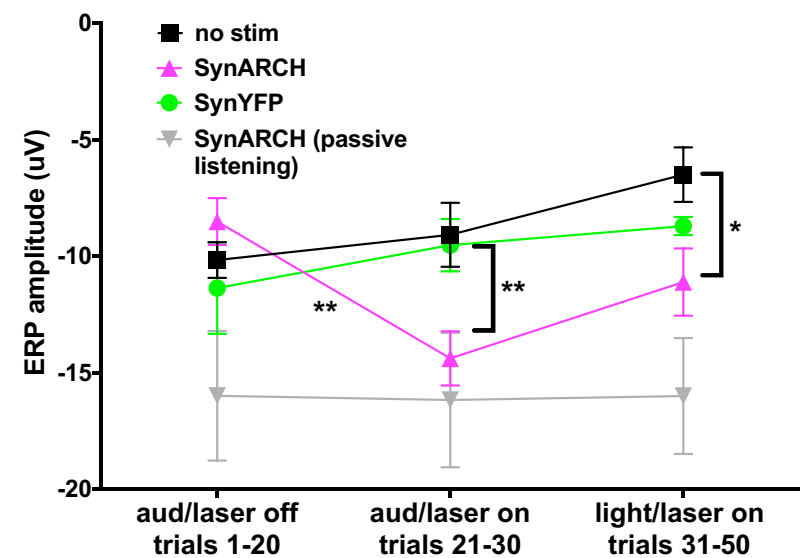

Figure 3. Inhibiting mPFC potentiates the N1 of the auditory ERP and disrupts auditory-visual rule switching. $A$, AAV-Syn-ARCH3.0-eYFP was injected bilaterally into the mPFC. $\boldsymbol{B}$, Schematic of the rule-switching task: mice first learned an auditory cue-based rule and then, after 30 trials, switched to a light cue-based rule. $C$, Optical inhibition of mPFC does not affect performance based on the established auditory rule but impairs switching to the new visual rule. ${ }^{* *} p<0.01$. D. Optical inhibition of $\mathrm{mPFC}$ results in perseverative errors during the rule switch (WT, $n=9 ;$ SynARCH, $n=$ $6 ;$ YFP, $n=4) .{ }^{* *} p<0.01$. E, Example auditory ERPs from one mouse showing that optical inhibition of mPFC modulates sensory processing of the relevant cue by increasing the N1 component of the auditory ERP. F, Quantification of $\mathrm{N} 1$ amplitude across the three task conditions in the presence or absence of optogenetic inhibition. Optogenetic inhibition of mPFC increases the $\mathrm{N} 1$ amplitude of the auditory ERP, yielding an amplitude similar to that observed during passive (nonattended) listening (WT, $n=6 ;$ SynARCH, $n=6 ; \mathrm{YFP}, n=4) .{ }^{*} p<0.05,{ }^{* *} p<0.01$. 
A

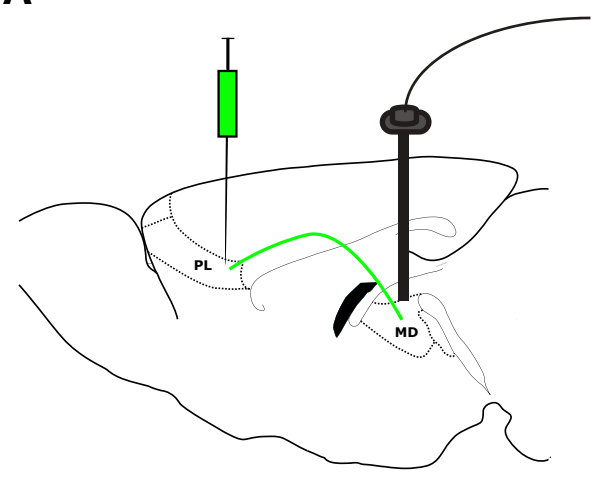

C

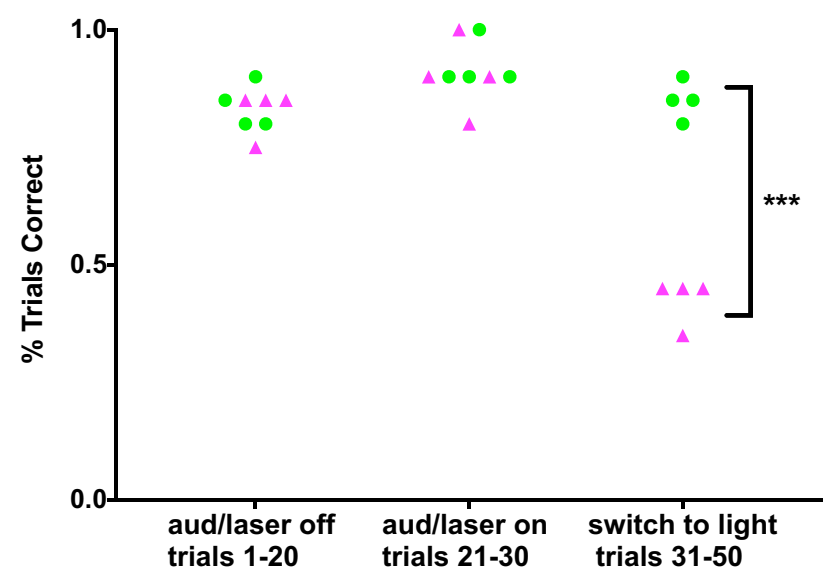

$\mathbf{E}$

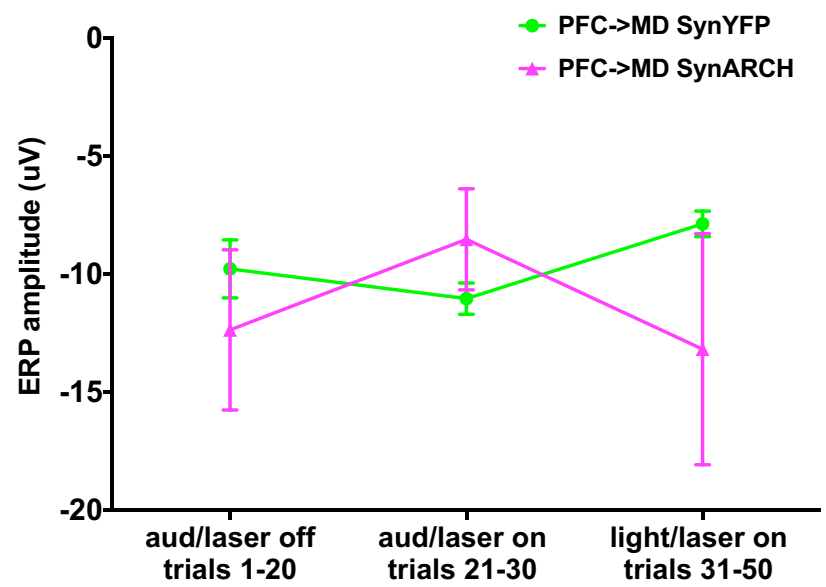

PFC->MD SynYFP

PFC->MD SynARCH
B

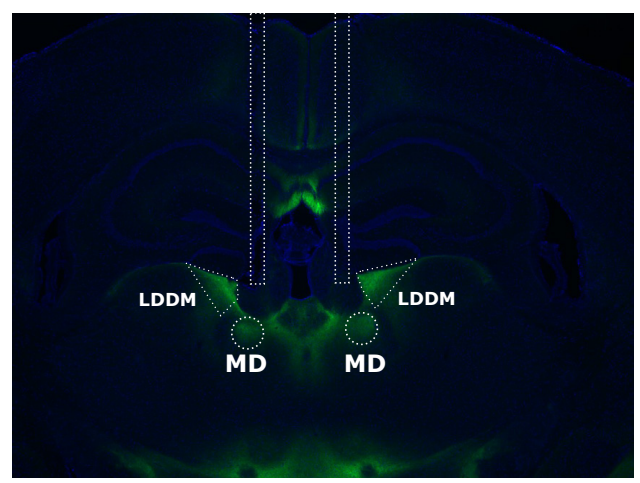

D

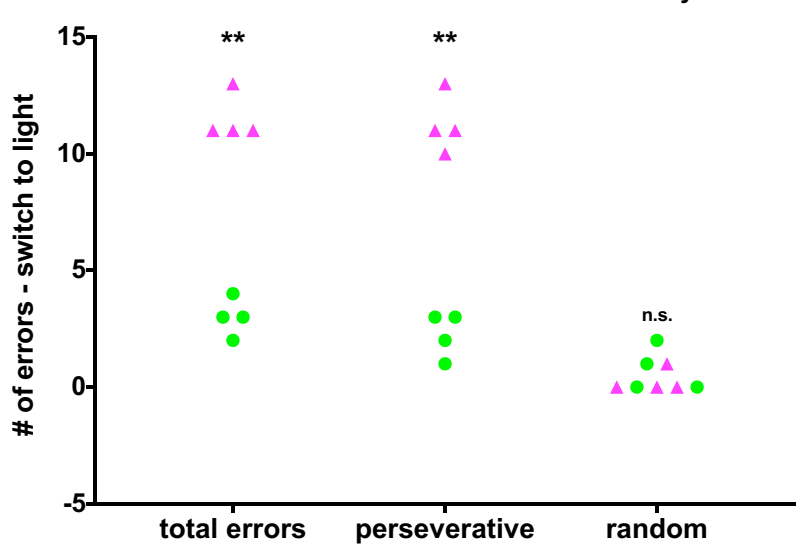

Figure 4. Optogenetic inhibition of $\mathrm{mPFC}$ projections to MD thalamus disrupts rule switching but has no effect on auditory ERPs. A, AAV-Syn-ARCH3.0-eYFP was injected bilaterally into mPFC, and optical fibers were placed bilaterally over MD thalamus. PL $=$ prelimbic cortex. $\boldsymbol{B}$, Histology showing mPFC projection terminals expressing Syn-ARCH3.0-eYFP in the MD thalamus as well as in the nearby laterodorsal dorsomedial thalamus (LDDM). The location of optical fibers has been outlined. C, Optical inhibition of mPFC projections to MD does not affect performance based on an established auditory rule but disrupts performance during the switch to a visual rule (SynARCH, $n=4 ; \mathrm{YFP}, n=4$ ). D, Optical inhibition of $\mathrm{mPFC}$ projections to MD results in perseverative errors during the attempt to switch to a visual rule. E, Quantification of the $\mathrm{N} 1$ amplitude of the auditory ERP across the three conditions. Optical inhibition of mPFC projections to MD had no significant effect on the $\mathrm{N} 1$ amplitude. ${ }^{* * *} p<0.001 ;{ }^{* *} p<0.01$. 
First, ERP increases are generally observed in experiments in which multiple stimuli compete for attention. In contrast, studies in which fewer stimuli compete for attention reproduce our observation that ERP amplitude decreases with task engagement (Otazu et al., 2009; Fritz et al., 2010). Indeed, in our study, there is just one auditory tone that (1) originates from just two possible locations, (2) is well above the detection threshold, and (3) persists throughout the trial (rather than being presented for a limited time). In other words, the detection of the auditory stimulus is not challenging in our paradigm. A second key difference is that in the study by Wimmer et al. (2015), mice are well trained. By contrast, we test mice as they learn to selectively use either auditory or visual cues to guide their behavior. Finally, in the work of Wimmer et al. (2015), switching between auditory and visual rules occurs on a trial-by-trial basis in response to a cue, whereas our switches are uncued and occur after blocks of 20-30 trials.

In summary, Wimmer et al. (2015) tested the ability of well trained mice to rapidly switch between difficult-to-detect cues. By contrast, we test the ability of mice to learn new rules involving easily detected cues. Thus, our task places lower demands on attention but higher demands on flexible learning. In some ways, these differences parallel those between human tasks that assess distinct aspects of executive function. For example, the study by Wimmer et al. (2015) resembles an explicitly cued, trial-by-trial switching Stroop task, in which the rules of the task are clearly laid out, but the stimuli are difficult to detect and require the rapid and flexible deployment of attention. In contrast, our task resembles the Wisconsin Card Sorting Task in the sense that the stimuli are easy to detect, but the rules of the task are implicit and must be learned while the task is being performed. In this framework, flexible behavior that is well learned and depends on the rapid deployment of attention may depend on "sensory selection" mediated by prefrontal control of gain in the sensory thalamus (Wimmer et al., 2015). By contrast, flexible behavior that reflects the acquisition of new rules seems to recruit prefrontal projections to MD thalamus without modulating sensory gain. In this case, when stimuli are easy to detect, flexibility does not seem to depend on sensory selection and, in fact, task engagement seems to drive decreased ERP amplitude.

Our finding that inhibiting mPFC-MD projections impairs certain forms of behavioral flexibility builds on previous work showing that inhibiting the MD thalamus impairs mPFC-MD interactions, measured by $\beta$-frequency synchronization, during working memory (Parnaudeau et al., 2013), as well as reversal learning (Parnaudeau et al., 2015). Notably, these previous studies chemogenetically inactivated the MD thalamus, whereas we used optogenetics to inhibit mPFC terminals in MD thalamus or mPFC neurons that project to MD thalamus. Thus, our results specifically and directly implicate projections from MPFC to MD in flexible behavior.

Importantly, to show that the mPFC-MD projections are important for behavioral flexibility, we used Arch to disrupt their function. Recent studies have confirmed that Arch powerfully suppresses action potential-evoked release from synaptic terminals (Mahn et al., 2016); this study found that Arch activation also increases action potential-independent spontaneous release from synaptic terminals. Action potential-evoked release generally elicits synaptic current at least an order of magnitude larger than those associated with action potential-independent spontaneous release. Regardless, these effects should disrupt the normal transmission of action potential-dependent information across this synapse. Thus, our finding that activating Arch in projections from the $\mathrm{mPFC}$ to $\mathrm{MD}$ thalamus prevents switching from an auditory to a visual cue-based rule without affecting the auditory ERP shows that these projections play a role in behavioral flexibility but do not necessarily contribute to task engagementdriven changes in ERP amplitude.

\section{Prefrontal circuits for cognitive flexibility}

Our previous work, using a task based on switching between odor and digging medium-based rules, showed that inhibiting prefrontal interneurons produces perseveration (Cho et al., 2015). Conversely, optogenetically stimulating prefrontal interneurons at gamma frequencies could rescue deficient cognitive flexibility in mutant mice. Gamma-frequency optogenetic stimulation strongly recruits parvalbumin (PV) interneuron-driven gamma rhythms (Cardin et al., 2009), and another study from our laboratory showed that prefrontal PV interneurons preferentially target L5 output neurons projecting to subcortical targets, e.g., MD thalamus (Lee et al., 2014). Thus, the form of stimulation that we found potently regulates cognitive flexibility also potently regulates mPFC-MD output, which we now show is required for flexible behavior. Another study from our laboratory also showed that these same subcortically projecting L5 neurons are selectively modulated by dopamine $\mathrm{D}_{2}$ receptors (Gee et al., 2012), which have also been implicated in flexible behavior (Floresco et al., 2006; St Onge et al., 2011). Together, these findings converge on a circuit in which PV interneurons, gamma oscillations, dopamine, and MD-projecting prefrontal neurons work together to enable flexible behavior. Importantly, although we observed increased prefrontal gamma oscillations during the auditory portion of our task, we have not fully worked out their precise function in this context and interpret them only as evidence for prefrontal engagement. Future studies may also examine whether other switching paradigms similarly depend on mPFC-MD output.

\section{The medial prefrontal cortex and task engagement}

Cortical LFP responses to auditory stimuli and auditory ERPs are suppressed when rats or ferrets perform a task, relative to when they hear the same tones in a task-free context (Otazu et al., 2009; Fritz et al., 2010). In mice, the responses of many excitatory neurons in auditory cortex are smaller when tones are presented during a task, compared with passive listening, and cholinergic modulation contributes to this process by depolarizing inhibitory neurons (Kuchibhotla et al., 2017). However, a similar effect, the recruitment of inhibitory interneurons and suppression of excitatory neurons in auditory cortex, is also observed during movement (Schneider et al., 2014). The latter effect appears to be driven by inputs to auditory cortex from secondary motor cortex, and another study has shown that secondary motor cortex is involved in flexible sensorimotor behavior (Siniscalchi et al., 2016). Here we show that auditory ERPs are suppressed when mice engage in a sensory cue-guided decision-making task and that this depends on the mPFC. Future studies will be needed to determine whether the $\mathrm{mPFC}$ modulates auditory cortical responses via a novel mechanism or one of the two mechanisms described above (mPFC connections to secondary motor cortex or to cholinergic neurons that project to auditory cortex). However, an important clue is that MPFC-auditory cortex theta synchronization increases when ERP suppression is observed, suggesting rapid time scale coupling between oscillatory mechanisms (such as inhibitory interneurons) in mPFC and auditory cortex. 


\section{Conclusion}

Here we show that a specific prefrontal output stream to MD thalamus regulates behavioral flexibility in a manner that can be dissociated from the top-down control of a marker of task engagement. Interestingly, reduced functional connectivity between the PFC and MD thalamus has been repeatedly linked to schizophrenia (Woodward et al., 2012; Anticevic et al., 2015). Thus, our results suggest that this specific pattern of thalamocortical disconnectivity may drive cognitive deficits that are at the core of schizophrenia (Green, 2006; Minzenberg and Carter, 2012). Future studies will elucidate how recurrent network interactions (Durstewitz et al., 2010; Mante et al., 2013), dopamine $\mathrm{D}_{2}$ receptors (Gee et al., 2012; Ellwood et al., 2017), and PV interneuron-driven gamma-frequency inhibition (Lee et al., 2014; Cho et al., 2015) can facilitate behavioral flexibility by regulating the PFC-MD output stream. Conversely, abnormalities in PV interneurons, gamma oscillations, and/or dopaminergic signaling may converge on the PFC-MD output stream, thereby driving cognitive deficits in conditions such as schizophrenia.

\section{References}

Amann LC, Gandal MJ, Halene TB, Ehrlichman RS, White SL, McCarren HS, Siegel SJ (2010) Mouse behavioral endophenotypes for schizophrenia. Brain Res Bull 83:147-161. CrossRef Medline

Anticevic A, Haut K, Murray JD, Repovs G, Yang GJ, Diehl C, McEwen SC, Bearden CE, Addington J, Goodyear B, Cadenhead KS, Mirzakhanian H, Cornblatt BA, Olvet D, Mathalon DH, McGlashan TH, Perkins DO, Belger A, Seidman LJ, Tsuang MT, et al. (2015) Association of thalamic dysconnectivity and conversion to psychosis in youth and young adults at elevated clinical risk. JAMA Psychiatry 72:882-891. CrossRef Medline

Birrell JM, Brown VJ (2000) Medial frontal cortex mediates perceptual attentional set shifting in the rat. J Neurosci 20:4320-4324. Medline

Bissonette GB, Martins GJ, Franz TM, Harper ES, Schoenbaum G, Powell EM (2008) Double dissociation of the effects of medial and orbital prefrontal cortical lesions on attentional and affective shifts in mice. J Neurosci 28:11124-11130. CrossRef Medline

Cardin JA, CarlénM, Meletis K, Knoblich U, Zhang F, Deisseroth K, Tsai LH, Moore CI (2009) Driving fast-spiking cells induces gamma rhythm and controls sensory responses. Nature 459:663-667. CrossRef Medline

Cho KK, Hoch R, Lee AT, Patel T, Rubenstein JL, Sohal VS (2015) Gamma rhythms link prefrontal interneuron dysfunction with cognitive inflexibility in Dlx5/6(+/-) mice. Neuron 85:1332-1343. CrossRef Medline

Durstewitz D, Vittoz NM, Floresco SB, Seamans JK (2010) Abrupt transitions between prefrontal neural ensemble states accompany behavioral transitions during rule learning. Neuron 66:438-448. CrossRef Medline

Ellwood IT, Patel T, Wadia V, Lee AT, Liptak AT, Bender KJ, Sohal VS (2017) Tonic or phasic stimulation of dopaminergic projections to prefrontal cortex causes mice to maintain or deviate from previously learned behavioral strategies. J Neurosci 37:8315-8329. CrossRef Medline

Featherstone RE, Phillips JM, Thieu T, Ehrlichman RS, Halene TB, Leiser SC, Christian E, Johnson E, Lerman C, Siegel SJ (2012) Nicotine receptor subtype-specific effects on auditory evoked oscillations and potentials. PLoS One 7:e39775. CrossRef Medline

Floresco SB, Magyar O, Ghods-Sharifi S, Vexelman C, Tse MT (2006) Multiple dopamine receptor subtypes in the medial prefrontal cortex of the rat regulate set-shifting. Neuropsychopharmacology 31:297-309. CrossRef Medline

Floresco SB, Block AE, Tse MT (2008) Inactivation of the medial prefrontal cortex of the rat impairs strategy set-shifting, but not reversal learning, using a novel, automated procedure. Behav Brain Res 190:85-96. CrossRef Medline

Fritz JB, David SV, Radtke-Schuller S, Yin P, Shamma SA (2010) Adaptive, behaviorally gated, persistent encoding of task-relevant auditory information in ferret frontal cortex. Nat Neurosci 13:1011-1019. CrossRef Medline
Gee S, Ellwood I, Patel T, Luongo F, Deisseroth K, Sohal VS (2012) Synaptic activity unmasks dopamine $\mathrm{D}_{2}$ receptor modulation of a specific class of layer V pyramidal neurons in prefrontal cortex. J Neurosci 32:49594971. CrossRef Medline

Green MF (2006) Cognitive impairment and functional outcome in schizophrenia and bipolar disorder. J Clin Psychiatry 67:e12. CrossRef Medline

Gregoriou GG, Rossi AF, Ungerleider LG, Desimone R (2014) Lesions of prefrontal cortex reduce attentional modulation of neuronal responses and synchrony in V4. Nat Neurosci 17:1003-1011. CrossRef Medline

Johnston K, Levin HM, Koval MJ, Everling S (2007) Top-down controlsignal dynamics in anterior cingulate and prefrontal cortex neurons following task switching. Neuron 53:453-462. CrossRef Medline

Kuchibhotla KV, Gill JV, Lindsay GW, Papadoyannis ES, Field RE, Sten TA, Miller KD, Froemke RC (2017) Parallel processing by cortical inhibition enables context-dependent behavior. Nat Neurosci 20:62-71. CrossRef Medline

Lee AT, Gee SM, Vogt D, Patel T, Rubenstein JL, Sohal VS (2014) Pyramidal neurons in prefrontal cortex receive subtype-specific forms of excitation and inhibition. Neuron 81:61-68. CrossRef Medline

Mahn M, Prigge M, Ron S, Levy R, Yizhar O (2016) Biophysical constraints of optogenetic inhibition at presynaptic terminals. Nat Neurosci 19:554556. CrossRef Medline

Mante V, Sussillo D, Shenoy KV, Newsome WT (2013) Context-dependent computation by recurrent dynamics in prefrontal cortex. Nature 503:78 84. CrossRef Medline

Minzenberg MJ, Carter CS (2012) Developing treatments for impaired cognition in schizophrenia. Trends Cogn Sci 16:35-42. CrossRef Medline

Otazu GH, Tai LH, Yang Y, Zador AM (2009) Engaging in an auditory task suppresses responses in auditory cortex. Nat Neurosci 12:646-654. CrossRef Medline

Parnaudeau S, O'Neill PK, Bolkan SS, Ward RD, Abbas AI, Roth BL, Balsam PD, Gordon JA, Kellendonk C (2013) Inhibition of mediodorsal thalamus disrupts thalamofrontal connectivity and cognition. Neuron 77 : 1151-1162. CrossRef Medline

Parnaudeau S, Taylor K, Bolkan SS, Ward RD, Balsam PD, Kellendonk C (2015) Mediodorsal thalamus hypofunction impairs flexible goaldirected behavior. Biol Psychiatry 77:445-453. CrossRef Medline

Prichard D, Theiler J (1994) Generating surrogate data for time series with several simultaneously measured variables. Phys Rev Lett 73:951-954. CrossRef Medline

Ragozzino ME, Detrick S, Kesner RP (1999) Involvement of the prelimbicinfralimbic areas of the rodent prefrontal cortex in behavioral flexibility for place and response learning. J Neurosci 19:4585-4594. Medline

Rodgers CC, DeWeese MR (2014) Neural correlates of task switching in prefrontal cortex and primary auditory cortex in a novel stimulus selection task for rodents. Neuron 82:1157-1170. CrossRef Medline

Schneider DM, Nelson A, Mooney R (2014) A synaptic and circuit basis for corollary discharge in the auditory cortex. Nature 513:189-194. CrossRef Medline

Siniscalchi MJ, Phoumthipphavong V, Ali F, Lozano M, Kwan AC (2016) Fast and slow transitions in frontal ensemble activity during flexible sensorimotor behavior. Nat Neurosci 19:1234-1242. CrossRef Medline

St Onge JR, Abhari H, Floresco SB (2011) Dissociable contributions by prefrontal $D_{1}$ and $D_{2}$ receptors to risk-based decision making. J Neurosci 31:8625-8633. CrossRef Medline

Wallis JD, Anderson KC, Miller EK (2001) Single neurons in prefrontal cortex encode abstract rules. Nature 411:953-956. CrossRef Medline

Wimmer RD, Schmitt LI, Davidson TJ, Nakajima M, Deisseroth K, Halassa MM (2015) Thalamic control of sensory selection in divided attention. Nature 526:705-709. CrossRef Medline

Woodward ND, Karbasforoushan H, Heckers S (2012) Thalamocortical dysconnectivity in schizophrenia. Am J Psychiatry 169:1092-1099. CrossRef Medline

Zhang S, Xu M, Kamigaki T, Hoang Do JP, Chang WC, Jenvay S, Miyamichi K, Luo L, Dan Y (2014) Selective attention. Long-range and local circuits for top-down modulation of visual cortex processing. Science 345:660 665. CrossRef Medline 